\title{
Prolonged treatment of COVID-19 pneumonia with high-flow nasal oxygen: A story of oxygen and resilience
}

\author{
G G Audley, ${ }^{1}$ MB ChB, Dip HIV Man (SA); P Frankenfeld, ${ }^{1,2}$ MB ChB, FCP (SA); S Dlamini, ${ }^{1,3}$ MB ChB, FCP (SA), Cert ID (SA) Phys; \\ P Raubenheimer, ${ }^{1}$ MB ChB, FCP (SA); J Hoare, ${ }^{4}$ MB ChB, MRC (Psych), FC Psych (SA), MPhil (Neuropsychiatry); \\ L Cairncross, ${ }^{5} \mathrm{MB}$ ChB, FCS (SA), MMed (Surg); R van den Berg, ${ }^{1} \mathrm{MB}$ ChB, DA (SA), Dip PEC (SA); M V Gule, ${ }^{1,6}$ MB ChB, FCP (SA); \\ G Calligaro, ${ }^{1,7}$ BSc Hons, MB ChB, Dip PEC (SA), MMed (Med), FCP (SA), Cert Pulm (SA) \\ ${ }^{1}$ Department of General Medicine, Faculty of Health Sciences, University of Cape Town and Groote Schuur Hospital, Cape Town, South Africa \\ 2 Division of Geriatric Medicine, Department of Medicine, Faculty of Health Sciences, University of Cape Town and Groote Schuur Hospital, \\ Cape Town, South Africa \\ ${ }^{3}$ Division of Infectious Diseases and HIV Medicine, Department of Medicine, Faculty of Health Sciences, University of Cape Town and Groote \\ Schuur Hospital, Cape Town, South Africa \\ ${ }^{4}$ Department of Psychiatry and Mental Health, University of Cape Town and Groote Schuur Hospital, Cape Town, South Africa \\ ${ }^{5}$ Division of Endocrine Surgery, Department of Surgery, Faculty of Health Sciences, University of Cape Town and Groote Schuur Hospital, \\ Cape Town, South Africa \\ ${ }^{6}$ Department of Neurology, Faculty of Health Sciences, University of Cape Town and Groote Schuur Hospital, Cape Town, South Africa \\ ${ }^{7}$ Division of Pulmonology, Department of Medicine and UCT Lung Institute, Faculty of Health Sciences, University of Cape Town, South Africa
}

Corresponding author: G G Audley (ggaudley@gmail.com)

The COVID-19 pandemic has placed significant strain on the oxygen delivery infrastructure of health facilities in resource-constrained health systems. In this case report, we describe a patient with severe COVID-19 pneumonia who was managed with high-flow nasal oxygen for 40 days, with an eventual successful outcome. We discuss the oxygen delivery infrastructure needed to offer this intervention, as well as the psychosocial impact on those undergoing treatment.

S Afr Med J 2020;110(12):1168-1171. https://doi.org/10.7196/SAMJ.2020.v110i12.15325

At the end of 2019, a novel coronavirus, SARS-CoV-2, resulted in an acute respiratory illness epidemic - COVID-19 - in Wuhan, China. ${ }^{[1]}$ The disease rapidly spread globally, and on 11 March 2020 the World Health Organization declared the outbreak a pandemic. ${ }^{[2]}$ Since identification of the first case of COVID-19 in South Africa (SA) on 5 March 2020, the number of confirmed cases as of 20 September 2020 totalled $659656 .^{[3,4]}$ One in five people with confirmed COVID-19 require hospitalisation. ${ }^{[5]}$ In most cases the indication for hospitalisation is hypoxaemia requiring supplemental oxygen therapy, the duration of which ranges from days to months, ${ }^{[6]}$ putting tremendous strain on hospital infrastructure, particularly central oxygen supply. Patients hospitalised with COVID-19 have varying levels of supplemental oxygen requirements. One strategy that has been employed to combat severe COVID-19 respiratory failure and hypoxaemia is high-flow nasal oxygen (HFNO).$^{[7]} \mathrm{HFNO}$ is a device that delivers $30-60 \mathrm{~L} / \mathrm{min}$ of heated and humidified air and oxygen blend at the desired fraction of inspired oxygen $\left(\mathrm{FiO}_{2}\right)$ via a wide-bore nasal cannula ${ }^{[8]}$ This method reduces anatomical dead space, work of breathing and respiratory rate, while increasing positive pressure and compliance. ${ }^{[8]}$

At Groote Schuur Hospital (GSH), a tertiary referral hospital in Western Cape Province, SA, 343 patients admitted with COVID-19 acute hypoxic respiratory failure from 7 May to 26 August 2020 have been treated with HFNO in dedicated COVID-19 high-care wards. With up to 34 HFNO machines simultaneously operational in these wards during the peak of the pandemic, and most patients requiring flow of $60 \mathrm{~L} / \mathrm{min}$ and $\mathrm{FiO}_{2}$ upwards of $90 \%$, sufficient and reliable central oxygen supply was, and continues to be, essential. Reliable central oxygen supply at healthcare facilities is a particular problem in resource-constrained sub-Saharan African settings, making the upscaling of HFNO as a potentially beneficial intervention challenging. ${ }^{[9]}$

In this report, we present a case of prolonged and ultimately successful treatment of COVID-19 pneumonia treated successfully with HFNO.

\section{Case report}

A 55-year-old, obese (BMI $32 \mathrm{~kg} / \mathrm{m}^{2}$ ) non-smoking woman, previously well, was referred to our tertiary hospital with COVID19 pneumonia. She had been diagnosed on a nasopharyngeal swab that was reverse transcriptase polymerase chain reaction-positive for SARS-CoV-2 after presenting to her local district hospital with a 1-week history of myalgia, fever, dry cough and progressive worsening shortness of breath. Fig. 1 shows the admission chest radiograph. Her only previous illness was pulmonary tuberculosis in childhood, successfully treated, and she had grown up in a rural settlement in Eastern Cape Province with significant indoor biomass fuel smoke exposure. Her referral to our hospital was triggered by increasing oxygen requirements over a 5 -day period, no longer maintaining saturations of $\sim 92 \%$ on pulse oximetry on a nonrebreather reservoir face mask.

On examination, the patient was in marked respiratory distress in the prone position, and unable to complete full sentences owing to breathlessness. Table 1 presents arterial blood gas measurements 
prior to HFNO and Table 2 admission vital signs. The admission team immediately transferred her to a dedicated COVID high-care ward, and initiated $\mathrm{HFNO}$ at $60 \mathrm{~L} / \mathrm{min}$ with $\mathrm{FiO}_{2}$ 95\%. She was reassessed closely for 6 hours, during which time her condition stabilised. Intermittent awake prone positioning continued (Table 2), and ongoing management was framed around five main areas: oxygenation, anticoagulation, steroid provision, management of complications, and multidisciplinary psychosocial support.

\section{Oxygenation}

The patient required HFNO for a total of 40 days, with additional standard oxygen support for a further 16 days. She elected to remain predominantly in the prone position for the first 32 days owing to profound desaturation with even the slightest movement, and required an $\mathrm{FiO}_{2}$ of $>90 \%$ for the first 20 days. At this point, it was possible to wean the $\mathrm{FiO}_{2}$ gradually over several days (Fig. 2 shows HFNO

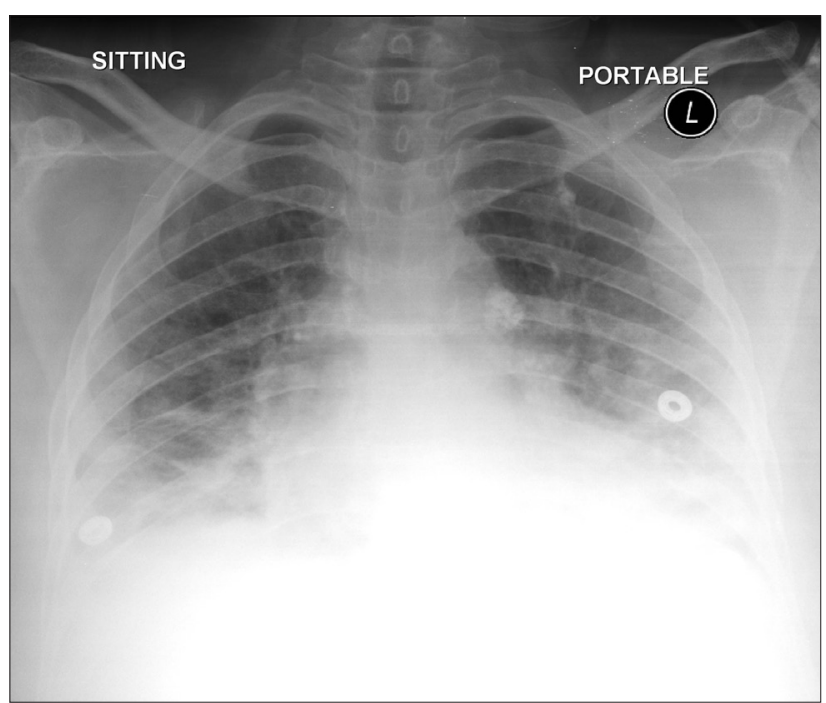

Fig. 1. Admission chest radiograph at the district hospital.

Table 1. Arterial blood gas measurements before HFNO (non-rebreather reservoir face mask at $15 \mathrm{~L} / \mathrm{min}$ )

\begin{tabular}{ll}
\hline $\mathrm{pH}$ & 7.523 \\
$\mathrm{pO}_{2}(\mathrm{kPa})$ & 2.78 \\
$\mathrm{pCO}_{2}(\mathrm{kPa})$ & 4.5 \\
Standard bicarbonate $(\mathrm{mmol} / \mathrm{L})$ & 28.3 \\
Lactate $(\mathrm{mmol} / \mathrm{L})$ & 1.8 \\
$\mathrm{P}: \mathrm{F}$ ratio $(\mathrm{mmHg})$ & 25 \\
& \\
$\mathrm{HFNO}=$ high-flow nasal oxygen; $\mathrm{pO}_{2}=\mathrm{partial}_{\text {pressure of oxygen; } \mathrm{pCO}_{2}=\text { partial pressure }}$ & \\
of carbon dioxide; $\mathrm{P}: \mathrm{F}$ ratio = arterial $\mathrm{pO}_{2} / \mathrm{FiO}_{2} ; \mathrm{FiO}_{2}=$ fraction of inspired oxygen.
\end{tabular}

settings over time). On day 36 of HFNO, she was able to mobilise from her bed to a chair for the first time. On day 40 of HFNO, she was successfully transitioned to a $40 \%$ Venturi face mask, on which she remained for a further 8 days, and thereafter transitioned to conventional nasal prong oxygen $\left(\mathrm{NPO}_{2}\right)$ at $4 \mathrm{~L} / \mathrm{min}$. After 8 days on $\mathrm{NPO}_{2}$ she was able to spend short periods of the day on room air alone. A full 56 days after admission, she was able to mobilise without supplementary oxygen.

\section{Anticoagulation}

Following admission to GSH, enoxaparin $1 \mathrm{mg} / \mathrm{kg}$ subcutaneously twice daily was continued. On day 25 on HFNO the patient was noted to have developed a spontaneous haematoma in her right thigh. The anti-factor Xa level was supratherapeutic at 1.87. Her enoxaparin dose was reduced according to serial anti-factor $\mathrm{Xa}$ levels. The surgical team was consulted, and the decision was made to manage the haematoma conservatively. The haematoma resolved without complication. Twice-daily enoxaparin at the adjusted dose was continued for the duration of her hospitalisation. In the last week of her hospitalisation, a computed tomography (CT) scan of her pulmonary arteries was performed to assess the presence of ongoing thrombosis to guide our anticoagulation strategy after discharge. The CT scan found no evidence of thromboembolic disease, only the typical bilateral, multifocal ground-glass opacification of COVID-19 pneumonia. The enoxaparin was subsequently stopped on discharge, with no outpatient anticoagulation prescribed.

\section{Steroids}

Dexamethasone $6 \mathrm{mg}$ intravenously daily was continued for 3 days after admission to GSH. On day 4 on HFNO, the intravenous cannula was removed, and prednisone $40 \mathrm{mg}$ orally daily was commenced. This dosage of prednisone was continued for the

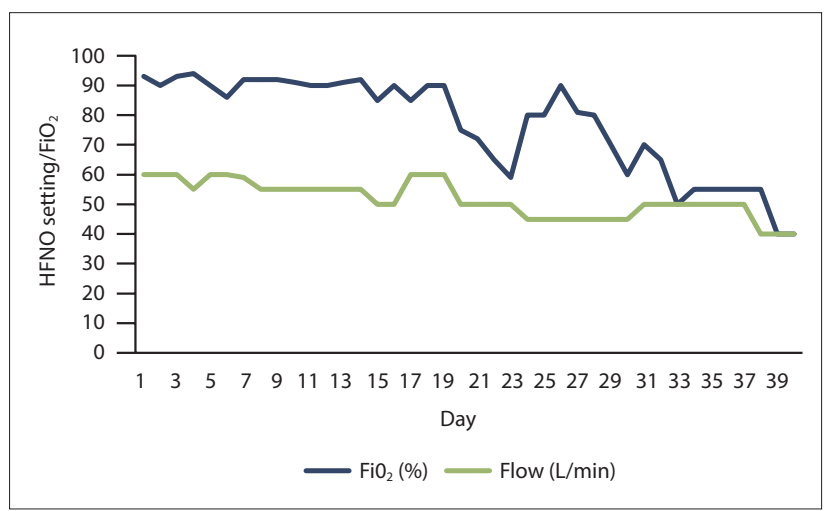

Fig. 2. HFNO settings over time. (HFNO = high-flow nasal oxygen; $\mathrm{FiO}_{2}=$ fraction of inspired oxygen.)

Table 2. Vital signs pre and post HFNO initiation at Groote Schuur Hospital

\begin{tabular}{|c|c|c|}
\hline & $\begin{array}{l}\text { Pre HFNO (non-rebreather reservoir } \\
\text { face mask at } 15 \mathrm{~L} / \mathrm{min} \text { ) (prone) }\end{array}$ & $\begin{array}{l}6 \text { hours post } \mathrm{HFNO} \text { initiation }\left(\mathrm{FiO}_{2} 93 \% \text {, }\right. \\
\text { flow rate } 60 \mathrm{~L} / \mathrm{min}) \text { (prone) }\end{array}$ \\
\hline Heart rate (bpm) & 120 & 115 \\
\hline Respiratory rate (breaths per minute) & 48 & 36 \\
\hline Peripheral oxygen saturation (\%) & 65 & 94 \\
\hline Temperature $\left({ }^{\circ} \mathrm{C}\right)$ & 37.8 & 37 \\
\hline Blood pressure (mmHg) & $136 / 77$ & $150 / 80$ \\
\hline ROX index & 1.59 & 2.81 \\
\hline
\end{tabular}


duration of her time on HFNO and subsequently gradually weaned to a stop by discharge.

\section{Management of complications}

On day 29 of HFNO, a deterioration in the patient's condition was noted. She developed a low-grade pyrexia of $37.4^{\circ} \mathrm{C}$, her respiratory rate climbed from 24 to 34 breaths per minute, and she developed a new-onset sinus tachycardia of $140 \mathrm{bpm}$. A thorough diagnostic evaluation for infection ensued. On enquiry, the patient only reported worsening breathlessness without any other specific symptomatology. Thorough examination revealed no obvious source of infection or COVID-19-related complications. The only remarkable feature elicited was new-onset bilateral coarse crackles upon lung auscultation. There were no clinical features of fluid overload. Urinalysis was unremarkable, a blood culture was drawn, and a chest radiograph showed diffuse, bilateral patchy groundglass opacities. Empirical antibiotics were initiated for suspected nosocomial pneumonia (piperacillin-tazobactam $4.5 \mathrm{~g}$ intravenously 6-hourly for 5 days and amikacin $1 \mathrm{~g}$ intravenously daily for 3 days). Blood culture and urine culture showed no growth. Her renal function remained normal, troponin $\mathrm{T}$ was not raised, and her anti-Xa level was therapeutic. Over the course of the next 3 days she recovered and completed the course of antibiotics.

\section{Multidisciplinary psychosocial support}

Multidisciplinary input was required to complete our comprehensive package of care. The patient was nursed in a high-care ward where a dedicated team of nursing staff worked tirelessly to care for her. Our dietetics team reviewed her regularly and provided daily nutritional supplementation. On day 30 on HFNO, she developed a number of mental health symptoms, including feeling sad, tired and overwhelmed, anxious and helpless. Prior to this she had demonstrated remarkable resilience despite the length of her hospital stay and the severity of her symptoms. Psychiatry provided ongoing counselling and advised the initiation of citalopram $20 \mathrm{mg}$ when her mental health symptoms, particularly her anxiety, negatively affected her HFNO weaning process. She responded well to the combination of citalopram and supportive counselling and thereafter was able to engage well with her transition to facemask oxygen. The social work team was also involved in managing her prolonged absence from work. Upon discharge, she was given a temporary disability grant to tide over the rest of her recovery.

Sixty-nine days after presenting to her local district hospital the patient was discharged home, mobilising freely without the need for supplemental oxygen. She received pneumococcal and influenza vaccines prior to discharge.

\section{Discussion}

The case described is remarkable in that it highlights incredible resilience on the part of the patient and dedication on the part of the treating multidisciplinary team. It is also striking to note the volume of oxygen consumed during her treatment course.

In a cohort of 293 patients with COVID-19 hypoxic respiratory failure enrolled at GSH and Tygerberg Hospital (TBH) during the pandemic, the median (interquartile range) time on HFNO in those successfully weaned was $6(3-9)$ days. ${ }^{[10]}$ The 40 days described above is the longest time any patient has spent on HFNO in this cohort - by a considerable margin. We have been unable to find another case of HFNO used for this duration before in a patient with a good outcome. There are no randomised trials of early v. late intubation for patients on HFNO, and we do not know whether this patient's dependence on oxygen would have been reduced with early intubation and ventilation. At GSH during the peak of the pandemic, the capacity to treat the numbers of patients with hypoxic respiratory failure with ventilation in the intensive care unit (ICU) was severely constrained. ${ }^{[1]]}$ HFNO was used as an alternative to try to overcome limited ICU resources. In the cohort from GSH and TBH, it was shown that in a resource-constrained setting, HFNO for COVID-19 hypoxic respiratory failure is feasible even outside the ICU and averts death or the need for mechanical ventilation in almost half of those who receive it. ${ }^{[10]}$

Over the course of our patient's 40 days on HFNO, she averaged an $\mathrm{FiO}_{2}$ of $77 \%$ at a flow rate of $52 \mathrm{~L} / \mathrm{min}$. This equates to the consumption of $40 \mathrm{~L}$ of gaseous oxygen per minute or a total of 2.3 million L of gaseous oxygen over the 40 days. Central oxygen is supplied in the liquid form. One litre of liquid oxygen weighs $1.14 \mathrm{~kg}$ and equates to $861 \mathrm{~L}$ of gaseous oxygen at one standard atmosphere and $21^{\circ} \mathrm{C} \cdot{ }^{[12]}$ Therefore, $2678 \mathrm{~L}$ or 3.05 tons of liquid oxygen was required to meet her oxygen needs while on HFNO. At our hospital prior to the COVID-19 pandemic, liquid oxygen was stored in two tanks, one holding 5 tons of liquid oxygen and the other 4 tons. In March 2020, in preparation for the anticipated surge in oxygen requirement, a larger 20-ton tank was installed (Fig. 3). During July 2020, at the peak of the COVID epidemic, oxygen consumption at GSH peaked at consumption of 8 tons of liquid oxygen a day.

Caring for this patient was significantly less costly, easier and associated with fewer complications than if she had been admitted to an ICU or ventilated. She was nursed in a six-bed cubicle in an open ward, with minimal extra staff allocated to her care. She was able to feed and prone herself, move her nasal interface, and use a bedpan with minimal assistance. Hospital complications for such an ill patient were minimal because of the use of HFNO - she was awake, developed no bedsores or other complications possible in

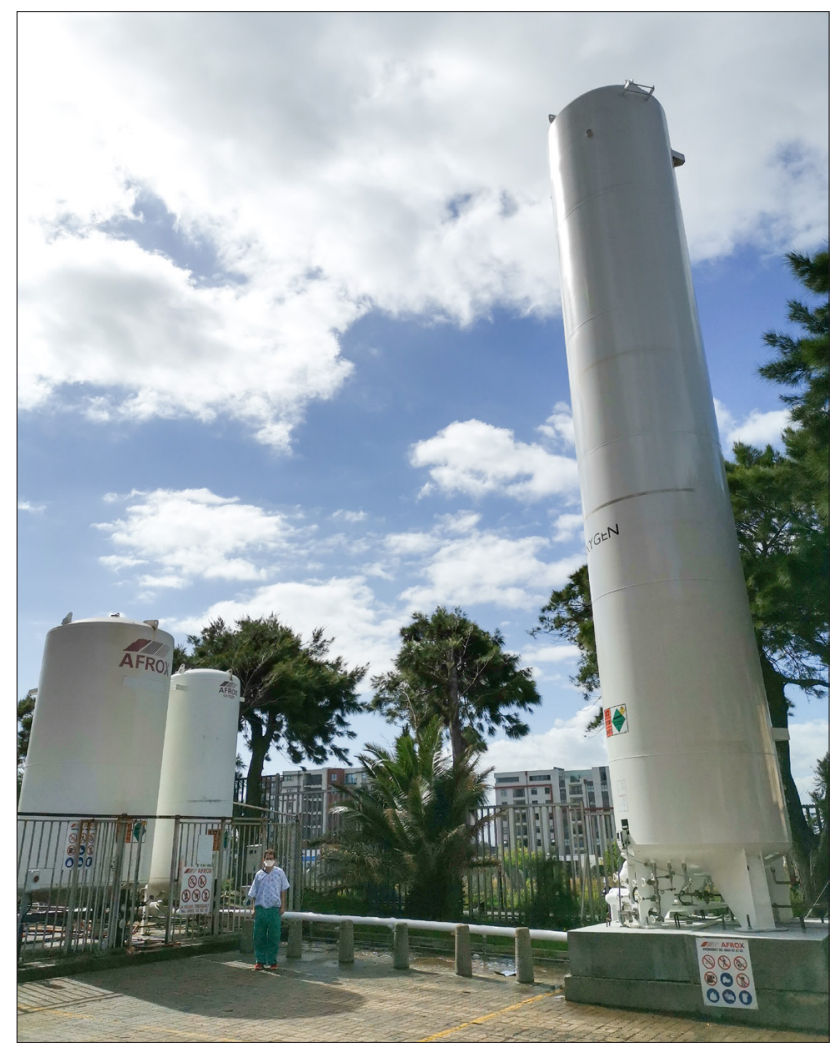

Fig. 3. Oxygen storage tanks at Groote Schuur Hospital. 
sedated patients, and required no ongoing intravenous access (with its possible complications of drip-site sepsis).

Despite the large amount of oxygen used, costs for this inpatient treatment were relatively low. Oxygen supply to GSH is currently priced at ZAR8.73/kg liquid oxygen. An approximated cost analysis of this patient's oxygen consumption equates to ZAR26 600 while on HFNO. Oxygen for healthcare use is a finite, albeit replenishable, resource. HFNO consumes oxygen to a greater extent than other methods of respiratory support, and facility assessment and preparation for increased demands should be considered when planning for COVID-19 hospital readiness. Ongoing assessment of on-site oxygen supplies and robustness of the oxygen supply chain should be a strategy to prevent and troubleshoot oxygen supply problems.

COVID-19 has rapidly become a global health emergency, resulting not only in physical health concerns but also psychological stress, as patients and families are exposed to the risk of premature death. Patients admitted to hospital with COVID-19 experience social isolation (particularly as families and visitors are not permitted in wards), physical discomfort, and fear of dying. The psychological impact of being surrounded by critically ill patients cannot be overstated. Over the 40-day period during which our patient was on HFNO, 183 other patients were initiated on HFNO in the high-care wards; $13 \%$ of this group died in the high-care wards, $35 \%$ were intubated, $40 \%$ were successfully weaned off HFNO and discharged, and the rest were still receiving HFNO at the time she was successfully weaned onto face-mask oxygen. It was incredibly challenging to be repeatedly exposed to fellow patients deteriorating around her, and perhaps even more challenging to see so many go home to their families while she was confined to the ward, dependent on oxygen. Despite these overwhelming circumstances, the patient showed remarkable resilience and ability to cope with stress. She enthusiastically used all the resources made available to her, communicated openly about her experience, and formed warm relationships with the multidisciplinary team. This is indeed a narrative of oxygen and resilience.
Declaration. None.

Acknowledgements. We acknowledge the tremendous contribution of the C13 ward staff, as well as the GSH COVID-19 response team, for what they have achieved in these challenging times.

Author contributions. GA and GC synthesised and collated the case report. All authors contributed to the management of this case. All authors contributed to writing and editing the manuscript.

Funding. None.

Conflicts of interest. None.

1. Guan W, Ni Z, Hu Y, et al. Clinical characteristics of coronavirus disease 2019 in China. N Engl J Med 2020;382(18):1708-1720. https://doi.org/10.1056/NEJMoa2002032

2. World Health Organization. Coronavirus disease pandemic. 10 April 2020. https://www.euro.who.int/ en/health-topics/health-emergencies/coronavirus-covid-19/novel-coronavirus-2019-ncov (accessed 2 July 2020).

3. European Centre for Disease Prevention and Control. COVID-19 situation update worldwide, as of 20 September 2020. 20 September 2020. https://www.ecdc.europa.eu/en/geographical-distribution2019 -ncov-cases (accessed 20 September 2020).

4. National Institute for Communicable Diseases. First case of COVID-19 coronavirus reported in SA. 5 March 2020. https://www.nicd.ac.za/first-case-of-covid-19-coronavirus-reported-in-sa/ (accessed 2 July 2020).

5. World Health Organization. Media statement: Knowing the risks of COVID-19. 8 March 2020. https:// www.who.int/indonesia/news/detail/08-03-2020-knowing-the-risk-for-covid-19 (accessed 2 July 2020). 6. Alhazzani W, Møller MH, Arabi YM, et al. Surviving Sepsis Campaign: Guidelines on the management of critically ill adults with coronavirus disease 2019 (COVID-19). Intensive Care Med 2020;46:854887. https://doi.org/10.1007/s00134-020-06022-5

7. Whittle JS, Pavlov I, Sacchetti AD, Atwood C, Rosenberg MS. Respiratory support for adult patients with COVID-19. J Am Coll Emerg Physicians Open 2020;1(2):95-101. https://doi.org/10.1002/ emp2.12071

8. Mauri T, Galazzi A, Binda F, et al. Impact of flow and temperature on patient comfort during respiratory support by high-flow nasal cannula. Crit Care 2018;22(1):1-8. https://doi.org/10.1186/ s13054-018-2039-4

9. Guardian. Fighting for breath: How the medical oxygen industry is failing African hospitals. 10 August 2020. https://www.theguardian.com/global-development/2020/aug/10/fighting-for-breath-how-the-

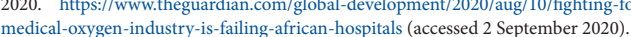

10. Calligaro GL, Lalla U, Audley G, et al. The utility of high-flow nasal oxygen for severe COVID-19 . Calligaro GL, Lalla $\mathrm{U}$, Audey G, et al. The utility of high-flow nasal oxygen for severe COVID-19
pneumonia in a resource-constrained setting: A multi-centre prospective observational study. pneumonia in a resource-constrained setting: A multi-centre prospective observational
EClinicalMedicine 2020 (epub 5 October 2020). https://doi.org/10.1016/j.eclinm.2020.100570

11. GroundUp. COVID-19: Groote Schuur on the brink. 21 May 2020. https://www.groundup.org.za/ article/covid-19-groote-schuur-brink/ (accessed 2 September 2020).

12. Air Products. Oxygen - weight and volume equivalents. 18 August 2020. http://www.airproducts. com/products/Gases/gas-facts/conversion-formulas/weight-and-volume-equivalents/oxygen.aspx (accessed 18 August 2020).

Accepted 1 October 2020. 